\title{
Some Induced Correlated Aggregating Operators with Interval Grey Uncertain Linguistic Information and Their Application to Multiple Attribute Group Decision Making
}

\author{
Zu-Jun Ma, Nian Zhang, and Ying Dai \\ School of Economic and Management, Southwest Jiaotong University, Chengdu, Sichuan 610031, China \\ Correspondence should be addressed to Nian Zhang; chinazhangnian@163.com
}

Received 3 November 2012; Accepted 30 December 2012

Academic Editor: Youqing Wang

Copyright (c) $2013 \mathrm{Zu}$-Jun Ma et al. This is an open access article distributed under the Creative Commons Attribution License, which permits unrestricted use, distribution, and reproduction in any medium, provided the original work is properly cited.

\begin{abstract}
We propose the interval grey uncertain linguistic correlated ordered arithmetic averaging (IGULCOA) operator and the induced interval grey uncertain linguistic correlated ordered arithmetic averaging (I-IGULCOA) operator based on the correlation properties of the Choquet integral and the interval grey uncertain linguistic variables to investigate the multiple attribute group decision making (MAGDM) problems, in which both the attribute weights and the expert weights are correlative. Firstly, the relative concepts of interval grey uncertain linguistic variables are defined and the operation rules between the two interval grey uncertain linguistic variables are established. Then, two new aggregation operators: the interval grey uncertain linguistic correlated ordered arithmetic averaging (IGULCOA) operator and the induced interval grey uncertain linguistic correlated ordered arithmetic averaging (I-IGULCOA) operator are developed and some desirable properties of the I-IGULCOA operator are studied, such as commutativity, idempotency, monotonicity, and boundness. Furthermore, the IGULCOA and I-IGULCOA operators based approach is developed to solve the MAGDM problems, in which both the attribute weights and the expert weights are correlative and the attribute values take the form of the interval grey uncertain linguistic variables. Finally, an illustrative example is given to verify the developed approach and to demonstrate its practicality and effectiveness.
\end{abstract}

\section{Introduction}

Recently, multiple attribute group decision making (MAGDM) has been extensively applied to various areas such as society, economics, management, and military. It is well known that the object things are complex and uncertain and human thinking is ambiguous. Hence, the majority of multiple attribute group decision making is also uncertain and fuzzy, and fuzziness is the major factor in the process of decision making. However, in dealing with the problem of incomplete information caused by poor information, decision making also demonstrates its greyness. The "fuzzy" means those uncertain factors in the evaluation information which are caused by the fuzziness of human thinking, while the "grey" means that objective uncertainty caused by the insufficient and incomplete information. Therefore, the "fuzzy" and the "grey" are different concepts, many scholars have studied the grey fuzzy multiple attribute decision making, which demonstrates not only its fuzziness, but also its greyness.

Since Zadeh introduced the concept of the fuzzy set [1] and Deng firstly presented the grey system theory [2], which were well applied in multiple attribute group decision making [3-13], the research on the grey fuzzy group decision making problem has been widely investigated and applied to a variety of fields. Chen [14] introduced the concept of the grey fuzzy in detail in his book. Bu and Zhang [15] presented an approach to transform the grey fuzzy number into the interval number, and then utilized the ranking method of interval number to rank the order of alternatives. Basing on the grey fuzzy multiple attribute decision making, in which both the fuzzy part and the grey part are real numbers, Jin and Lou [16, 17] used the decision making model which utilized the hamming distance to measure the alternatives and utilized 
the difference between the fuzzy positive ideal solution and the negative ideal solution to rank the order. In order to solve the grey fuzzy decision making, Dang and Sifeng [18] developed the maximum entropy formulism to determine attribute weight and ranked the order of alternatives based on the linear combination of fuzzy information and grey information. Meng et al. [19] proposed the grey degree and fuzzy degree with the interval numbers, and then, based on this, the mathematical model of interval-valued grey fuzzy comprehensive evaluation was established. At last its application to the selection of the preferred project was given. In many real-life decision making problems, the linguistic variable is easier to express fuzzy information and closer to actual condition; the research on linguistic decision making has witnessed rich achievements [3,11,20-27]. Liu and Jin [4] defined the concept of the interval grey linguistic variable where the fuzzy part and the grey part took the form of the uncertain linguistic variable and the interval number, respectively, studied the operation rules, and developed the multiple attribute decision making method based on the interval grey linguistic variable. Liu and Zhang [5] proposed the interval grey linguistic variables weighted geometric aggregation (IGLWGA) operator, and the interval grey linguistic variables ordered weighted geometric aggregation (IGLOWGA) operator and the interval grey linguistic variables hybrid weighted geometric aggregation (IGLHWGA) operator and then suggested a method for solving multiple attribute group decision making based on those operators. Zhang and Wei [28] introduced the interval grey linguistic variables ordered weighted aggregation (IGLOWA) operator, and then used the Choquet integral to develop the interval grey linguistic correlated ordered arithmetic aggregation (IGLCOA) operator and the interval grey linguistic correlated ordered geometric aggregation (IGLCOGA) operator. Those operators not only consider the importance of the elements, but also can reflect the correlations among the elements. Then, they developed an approach to multiple attribute decision making problems with correlative weights where the attribute values are given in terms of interval grey linguistic variables information based on those operators.

The existing grey fuzzy multiple attribute group decision making only considers the situation where all the elements in the grey fuzzy set are independent. However, in many practical situations, the elements in the grey fuzzy set are usually correlative. Therefore, we need to find some new ways to deal with the situations, in which the decision data in question are correlative and the weights are correlative. The Choquet integral [29] is a very useful way of measuring the expected utility of an uncertain event and can be utilized to depict the correlations of the decision data under consideration. Yager $[30,31]$ introduced the idea of order-induced aggregation to the Choquet aggregation operator and defined an induced Choquet ordered weighted averaging (C-OWA) operator, which allowed the ordering of the arguments to be based upon some other associated variables instead of ordering the arguments based on their values. Tan and Chen [32] developed the induced Choquet ordered averaging (I-COA) operator and applied it to aggregate fuzzy preference relations in group decision making. Xu [25] utilized the Choquet integral to propose the interval-valued intuitionistic fuzzy correlated averaging (IVIFCA) operator and the intervalvalued intuitionistic fuzzy correlated geometric (IVIFCG) operator to aggregate interval-valued intuitionistic fuzzy information and applied them to a practical decision making problem involving the prioritization of information technology improvement projects. Wei and Zhao [33] developed the induced intuitionistic fuzzy correlated averaging (I-IFCA) operator and induced intuitionistic fuzzy correlated geometric (I-IFCG) operator and developed to solve the MAGDM problems, in which both the attribute weights and the expert weights are usually correlative and attribute values take the form of intuitionistic fuzzy values.

Motivated by the correlation properties of the Choquet integral and the uncertain linguistic variables, in this paper, we propose the interval grey uncertain linguistic correlated ordered arithmetic averaging (IGULCOA) operator and the induced interval grey uncertain linguistic correlated ordered arithmetic averaging (I-IGULCOA) operator with interval grey uncertain linguistic variables information. The prominent characteristic of those operators is that they cannot only consider the importance of the elements or their ordered positions, but also reflect the correlations among the elements or their ordered positions. And we introduce those induced correlated aggregating operators to deal with group decision making problems. The aim of this paper is to investigate the MAGDM problems, in which both the attribute weights and the expert weights are correlative and the attribute values take the form of interval grey uncertain linguistic variables. In order to do so, the remainder of this paper is set out as follows. In the next section, we introduce some basic concepts related to interval grey uncertain linguistic variables and some operational laws of interval grey uncertain linguistic variables. In Section 3, we have developed two interval grey uncertain linguistic correlated aggregation operators: the IGULCOA operator and the I-IGULCOA operator. In Section 4, we have developed an approach to multiple attribute group decision making, in which both the attribute weights and the expert weights are correlative and the attribute values take the form of interval grey uncertain linguistic variables based on the IGULCOA operator and the I-IGULCOA operator with interval grey uncertain linguistic variables information. In Section 5, an illustrative example is pointed out. In Section 6, we conclude the paper and give some remarks.

\section{Preliminaries}

In this section, we briefly review some basic concepts to be used throughout the paper.

The linguistic approach is an approximate technique, which represents qualitative aspects as linguistic values by means of linguistic variables $[34,35]$.

Suppose that $S=\left\{s_{i} \mid i=-t, \ldots,-1,0,1, \ldots, t\right\}$ is a finite and totally ordered discrete term set, whose cardinality value is odd [35]. Any label $s_{i}$ represents a possible value for a linguistic variable (as shown in Figure 1), and it has the following characteristics: (1) the set is ordered as $s_{\alpha}>s_{\beta}$, if $\alpha>\beta$, and (2) there is the negative operator: $\operatorname{neg}\left(s_{\alpha}\right)=s_{-\alpha}$. 


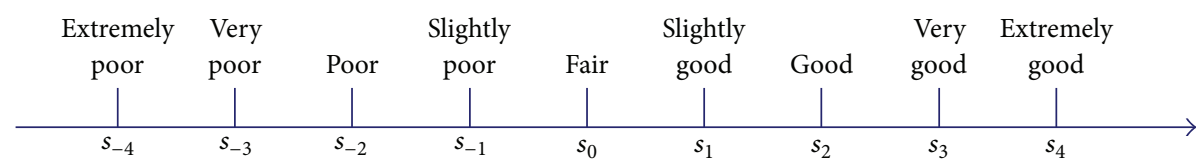

FIgURE 1: The linguistic labels.

We call this linguistic label set $S$ the additive linguistic scale. For example, a set of nine terms $S$ could be defined as follows:

$$
\begin{gathered}
S=\left\{s_{-4}=\text { extremely poor, } s_{-3}=\text { very poor, } s_{-2}=\right.\text { poor, } \\
s_{-1}=\text { slightly poor, } s_{0}=\text { fair }, s_{1}=\text { slightly good, } \\
\left.s_{2}=\text { good, } s_{3}=\text { very good, } s_{4}=\text { extremely good }\right\}
\end{gathered}
$$

in which $s_{i}<s_{j}$ if $i<j$.

To preserve all the given information, we extend the discrete term set $S$ to a continuous term set $S=\left\{s_{\alpha} \mid \alpha \epsilon\right.$ $[-t, t]\}$. If $s_{\alpha}<S$, then we call $s_{\alpha}$ an original linguistic term; otherwise, we call $s_{\alpha}$ a virtual linguistic term. In general, the decision maker uses the original linguistic terms to evaluate alternatives, and the virtual linguistic terms can only appear in operation $[10,36]$.

Let $\widetilde{s}=\left[s_{\alpha}, s_{\beta}\right]$, where $s_{\alpha}, s_{\beta} \in S, s_{\alpha}$ and $s_{\beta}$ are the lower and the upper limits, respectively, we then call $s$ an uncertain linguistic variable. Let $S$ be the set of all the uncertain linguistic variables [10].

Consider any three uncertain linguistic variables $\widetilde{s}=$ $\left[s_{\alpha}, s_{\beta}\right], \widetilde{s}_{1}=\left[s_{\alpha_{1}}, s_{\beta_{1}}\right]$, and $\widetilde{s}_{2}=\left[s_{\alpha_{2}}, s_{\beta_{2}}\right]$, then we define the operations $\widetilde{s}_{1} \oplus \widetilde{s}_{2}$ and $\lambda \widetilde{s}$ as follows:

(1) $\widetilde{s}_{1} \oplus \widetilde{s}_{2}=\left[s_{\alpha_{1}}, s_{\beta_{1}}\right] \oplus\left[s_{\alpha_{2}}, s_{\beta_{2}}\right]=\left[s_{\alpha_{1}} \oplus s_{\alpha_{2}}, s_{\beta_{1}} \oplus s_{\beta_{2}}\right]=$ $\left[s_{\alpha_{1}+\alpha_{2}}, s_{\beta_{1}+\beta_{2}}\right]$;

(2) $\lambda \widetilde{s}=\lambda\left[s_{\alpha}, s_{\beta}\right]=\left[s_{\lambda \alpha}, s_{\lambda \beta}\right]$, where $\lambda \in[0,1]$;

(3) $\widetilde{s}_{1} \oplus \widetilde{s}_{2}=\widetilde{s}_{2} \oplus \widetilde{s}_{1}$;

(4) $\lambda\left(\widetilde{s}_{1} \oplus \widetilde{s}_{2}\right)=\lambda \widetilde{s}_{1} \oplus \lambda \widetilde{s}_{2}$, where $\lambda \in[0,1]$;

(5) $\left(\lambda_{1}+\lambda_{2}\right) \widetilde{s}=\lambda_{1} \widetilde{s} \oplus \lambda_{2} \widetilde{s}$, where $\lambda_{1}, \lambda_{2} \in[0,1]$.

Definition 1 (see [14]). Let $\widetilde{A}(x)$ be the fuzzy subset in the space $X=\{x\}$; if the membership degree $\mu_{A}(x)$ of $x$ to $\widetilde{A}(x)$ is the grey in the interval $[0,1]$, and its grey is $v_{A}(x)$, then $\widetilde{A}(x)$ is called the grey fuzzy set in space $X$ (GF set, for short), denoted by $\underset{\otimes}{\widetilde{A}}(x)$, as follows:

$$
\underset{\otimes}{\widetilde{A}}(x)=\left\{\left(x, \mu_{A}(x), v_{A}(x)\right) \mid x \in X\right\} .
$$

The set pair mode is $\underset{\otimes}{\widetilde{A}}(x)=(\widetilde{A}(x), \underset{\otimes}{A}(x))$, where $\widetilde{A}(x)=$ $\left\{\left(x, \mu_{A}(x)\right) \mid x \in X\right\}$ is called the fuzzy part of $\underset{\otimes}{A}(x)$, and $\underset{\otimes}{A}(x)=\left\{\left(x, v_{A}(x)\right) \mid x \in X\right\}$ is called the grey part of $\underset{\otimes}{A}(x)$. So the grey fuzzy set is regarded as the generalization of the fuzzy set and the grey set.

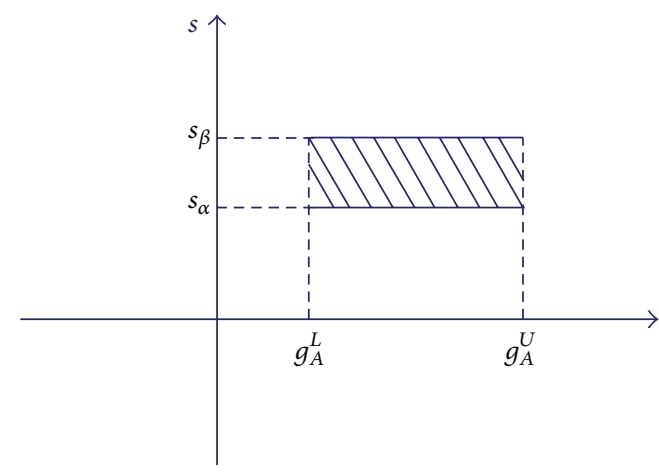

FIgURE 2: The interval grey linguistic variable.

Definition 2. Let $\underset{\otimes}{\widetilde{A}}(x)=(\widetilde{A}(x), \underset{\otimes}{A}(x))$ be the grey fuzzy number; if its fuzzy part is an uncertain linguistic variable $\widetilde{\mathcal{s}}=$ $\left[s_{\alpha}, s_{\beta}\right]$, where $s_{\alpha}, s_{\beta} \in S$, where $S$ is a finite and totally ordered discrete term set, and its grey part $\underset{\otimes}{A}(x)$ is a closed interval $\left[g_{A}^{L}, g_{A}^{U}\right]$, then $\underset{\otimes}{\widetilde{A}}(x)$ is called the interval grey uncertain linguistic variable, as shown in Figure 2.

Suppose that $\underset{\otimes}{\widetilde{A}}(x)=\left(\widetilde{s}_{1},\left[g_{A}^{L}, g_{A}^{U}\right]\right), \underset{\otimes}{\widetilde{B}}(x)=\left(\widetilde{s}_{2},\left[g_{B}^{L}, g_{B}^{U}\right]\right)$ are two interval grey uncertain linguistic variables, where $\widetilde{s}_{1}=$ $\left[s_{\alpha_{1}}, s_{\beta_{1}}\right]$ and $\widetilde{s}_{2}=\left[s_{\alpha_{2}}, s_{\beta_{2}}\right]$. The continuous ordered weighted averaging ((C-OWA), for short) operator which is developed by Yager [37] can be usefully applied to aggregate the grey part, the greyness of the grey part would be transformed into a real number, and then the fuzzy part integrates with the grey part, that is to say, the size of the interval uncertain grey linguistic variables can be gotten through comparing the size of $\left[s_{\alpha_{1}}, s_{\beta_{1}}\right] \times f_{\rho}\left(\left[\left(1-g_{A}^{U}\right),\left(1-g_{A}^{L}\right)\right]\right)$ and $\left[s_{\alpha_{2}}, s_{\beta_{2}}\right] \times$ $f_{\rho}\left(\left[\left(1-g_{B}^{U}\right),\left(1-g_{B}^{L}\right)\right]\right)$. Assume the ordering value $\widetilde{Q}(\underset{\otimes}{\widetilde{A}}(x))=$ $\left[s_{\alpha_{1}}, s_{\beta_{1}}\right] \times f_{\rho}\left(\left[\left(1-g_{A}^{U}\right),\left(1-g_{A}^{L}\right)\right]\right)$ and $\widetilde{Q}(\underset{\otimes}{\widetilde{B}}(x))=\left[s_{\alpha_{2}}, s_{\beta_{2}}\right] \times$ $f_{\rho}\left(\left[\left(1-g_{B}^{U}\right),\left(1-g_{B}^{L}\right)\right]\right)$, which can be obtained based on the continuous ordered weighted averaging (C-OWA) operator, such as $f_{p}([a, b])=\int_{0}^{1}(d \rho(y) / d y)(b-y(b-a)) d y$. In order to compare uncertain linguistic variables, we use the degree of possibility.

The function $\rho$ is denoted as basic unit-interval monotonic (BUM) functions. If $(\delta \geq 0)$, then $\rho(y)=y^{\delta}$.

Definition 3 (see [10]). Let $\widetilde{s}_{1}=\left[s_{\alpha_{1}}, s_{\beta_{1}}\right]$ and $\widetilde{s}_{2}=\left[s_{\alpha_{2}}, s_{\beta_{2}}\right]$ be two uncertain linguistic variables, and let len $\left(\widetilde{s}_{1}\right)=\beta_{1}-\alpha_{1}$ 
and len $\left(\widetilde{s}_{2}\right)=\beta_{2}-\alpha_{2}$, then the degree of possibility of $\widetilde{s}_{1} \geq \widetilde{s}_{2}$ is defined as follows:

$$
\begin{aligned}
p\left(\widetilde{s}_{1}\right. & \left.\geq \widetilde{s}_{2}\right) \\
& =\frac{\operatorname{Max}\left\{0, \operatorname{len}\left(\widetilde{s}_{1}\right)+\operatorname{len}\left(\widetilde{s}_{2}\right)\right\}-\operatorname{Max}\left(\beta_{2}-\alpha_{1}, 0\right)}{\operatorname{len}\left(\widetilde{s}_{1}\right)+\operatorname{len}\left(\widetilde{s}_{2}\right)} .
\end{aligned}
$$

Particularly, if both the uncertain linguistic variables $\widetilde{s}_{1}$ and $\widetilde{s}_{2}$ express precise information (i.e., if $\operatorname{len}\left(\widetilde{s}_{1}\right)+\operatorname{len}\left(\widetilde{s}_{2}\right)$ ), then we define the degree of possibility of $\widetilde{s}_{1} \geq \widetilde{s}_{2}$ as follows:

$$
p\left(\widetilde{s}_{1} \geq \widetilde{s}_{2}\right)= \begin{cases}1 & \text { if } \widetilde{s}_{1}>\widetilde{s}_{2} \\ \frac{1}{2} & \text { if } \widetilde{s}_{1}=\widetilde{s}_{2} \\ 0 & \text { if } \widetilde{s}_{1}<\widetilde{s}_{2} .\end{cases}
$$

The operation rules of ranking are defined as follows.

(1) If $P(\widetilde{Q}(\underset{\otimes}{\widetilde{A}}(x))>\widetilde{Q}(\underset{\otimes}{\widetilde{B}}(x)))=1$, then we have $\underset{\otimes}{\widetilde{A}}(x)>$ $\underset{\otimes}{\widetilde{B}}(x)$.

(2) If $P(\widetilde{Q}(\underset{\otimes}{\widetilde{A}}(x))>\widetilde{Q}(\underset{\otimes}{\widetilde{B}}(x)))=0$, then we have $\underset{\otimes}{\widetilde{A}}(x)<$ $\widetilde{B}(x)$.

(3) If $P(\widetilde{Q}(\underset{\otimes}{\widetilde{A}}(x))>\widetilde{Q}(\underset{\otimes}{\widetilde{B}}(x)))=1 / 2$ and $P\left(\widetilde{s}_{1}>\widetilde{s}_{2}\right)=1$, then we have $\underset{\otimes}{\widetilde{A}}(x)>\underset{\otimes}{\widetilde{B}}(x)$.

(4) If $P(\widetilde{Q}(\underset{\otimes}{\widetilde{A}}(x))>\widetilde{Q}(\underset{\otimes}{\widetilde{B}}(x)))=1 / 2$ and $P\left(\widetilde{s}_{1}>\widetilde{s}_{2}\right)=1 / 2$, then we have $\underset{\otimes}{\widetilde{A}}(x)=\underset{\otimes}{\widetilde{B}}(x)$.

(5) If $P(\widetilde{Q}(\underset{\otimes}{\widetilde{A}}(x))>\widetilde{Q}(\underset{\otimes}{\widetilde{B}}(x)))=1 / 2$ and $P\left(\widetilde{s}_{1}>\widetilde{s}_{2}\right)=0$, then we have $\underset{\otimes}{\widetilde{A}}(x)<\underset{\otimes}{\widetilde{B}}(x)$.

The operation rules of the interval grey uncertain linguistic variables are defined as follows:

(1) $\underset{\otimes}{\widetilde{A}}(x) \oplus \underset{\otimes}{\widetilde{B}}(x)=\left(\widetilde{s}_{1} \oplus \widetilde{s}_{2},\left[\left(1-\left(1-g_{A}^{L}\right) \times\left(1-g_{B}^{L}\right)\right),(1-\right.\right.$ $\left.\left.\left.\left(1-g_{A}^{U}\right) \times\left(1-g_{B}^{U}\right)\right)\right]\right)$;

(2) $\lambda \underset{\otimes}{\widetilde{A}}(x)=\left(\lambda \widetilde{s}_{1},\left[g_{A}^{L}, g_{A}^{U}\right]\right)$.

Definition 4. An IGULWAA operator of dimension $n$ is a function IGULWAA : $\Omega^{n} \rightarrow \Omega$, which has associated a set of weights or weighting vector $w=\left(\omega_{1}, \omega_{2}, \ldots, \omega_{n}\right)$ with $\omega_{j} \in[0,1], \sum_{j=1}^{n} \omega_{j}=1$, and is defined to aggregate a list of values $\left\{\underset{\otimes}{\widetilde{A}}\left(x_{1}\right), \underset{\otimes}{\widetilde{A}}\left(x_{2}\right), \ldots, \underset{\otimes}{\widetilde{A}}\left(x_{n}\right)\right\}$, where $\underset{\otimes}{\widetilde{A}}\left(x_{j}\right)=$ $\left(\left[s_{\alpha_{j}}, s_{\beta_{j}}\right],\left[g_{j}^{L}, g_{j}^{U}\right]\right)$. According to the following expression.

$$
\begin{aligned}
& \operatorname{IGULWAA}\left(\underset{\otimes}{\widetilde{A}}\left(x_{1}\right), \underset{\otimes}{\widetilde{A}}\left(x_{2}\right), \ldots, \underset{\otimes}{\widetilde{A}}\left(x_{n}\right)\right)=\bigoplus_{j=1}^{n} \omega_{j} \widetilde{A}\left(x_{j}\right) \\
& =\left(\left[s_{\sum_{j=1}^{n} \omega_{j} \alpha_{j}} s_{\sum_{j=1}^{n} \omega_{j} \beta_{j}}\right],\left[1-\prod_{j=1}^{n}\left(1-g_{j}^{L}\right), 1-\prod_{j=1}^{n}\left(1-g_{j}^{U}\right)\right]\right) .
\end{aligned}
$$

Definition 5. An IGULOWA operator of dimension $n$ is a function IGULOWA : $\Omega^{n} \rightarrow \Omega$, which has associated a set of weights or weighting vector $w=\left(\omega_{1}, \omega_{2}, \ldots, \omega_{n}\right)$ with $\omega_{j} \in[0,1], \sum_{j=1}^{n} \omega_{j}=1$, and is defined to aggregate a list of values $\left\{\underset{\otimes}{\widetilde{A}}\left(x_{1}\right), \underset{\otimes}{\widetilde{A}}\left(x_{2}\right), \ldots, \underset{\otimes}{\widetilde{A}}\left(x_{n}\right)\right\}$, where $\underset{\otimes}{\widetilde{A}}\left(x_{j}\right)=$ $\left(\left[\widetilde{s}_{\alpha_{j}}, \widetilde{s}_{\beta_{j}}\right],\left[g_{j}^{L}, g_{j}^{U}\right]\right)$. According to the following expression,

$$
\begin{aligned}
& \operatorname{IGULOWA}\left(\underset{\otimes}{\widetilde{A}}\left(x_{1}\right), \underset{\otimes}{\widetilde{A}}\left(x_{2}\right), \ldots, \underset{\otimes}{\widetilde{A}}\left(x_{n}\right)\right)=\bigoplus_{j=1}^{n} \omega_{j} \widetilde{A}\left(x_{\tau(j)}\right) \\
& =\left(\left[s_{\sum_{j=1}^{n} \omega_{j} \alpha_{\tau(j)}}, s_{\sum_{j=1}^{n} \omega_{j} \beta_{\tau(j)}}\right],\right. \\
& \left.\left[1-\prod_{j=1}^{n}\left(1-g_{\tau(j)}^{L}\right), 1-\prod_{j=1}^{n}\left(1-g_{\tau(j)}^{U}\right)\right]\right),
\end{aligned}
$$

where there is a permutation such that $\underset{\otimes}{\widetilde{A}}\left(x_{\tau(1)}\right) \geq \underset{\otimes}{\widetilde{A}}\left(x_{\tau(2)}\right) \geq$ $\cdots \geq \underset{\otimes}{\widetilde{A}}\left(x_{\tau(n)}\right)$, that is, $\underset{\otimes}{\widetilde{A}}\left(x_{\tau(j)}\right)$ the $j$ th largest value in the set $\left\{\underset{\otimes}{\widetilde{A}}\left(x_{1}\right), \underset{\otimes}{\widetilde{A}}\left(x_{2}\right), \ldots, \underset{\otimes}{\widetilde{A}}\left(x_{n}\right)\right\}$.

\section{Some Interval Grey Uncertain Linguistic Correlated Ordered Arithmetic Averaging Operators}

In multiple attribute group decision making, the considered attributes usually have different levels of importance and, thus, need to be assigned different weights. Some operators have been introduced to aggregate the interval grey uncertain linguistic variables together with independent weighted elements, but they only consider the addition of the importance of individual elements. However, in some practical situations, the elements in the interval grey uncertain linguistic variables have some correlations with each other and, thus, it is necessary to consider this issue. For real decision making problems, there is always some degree of interdependent characteristics between attributes. Usually, there is interaction among attributes of decision makers. However, this assumption is too strong to match decision behaviors in the real world. The independence axiom generally cannot be satisfied. Thus, it is necessary to consider this issue.

Let $m\left(x_{i}\right)(i=1,2, \ldots, n)$ be the weight of the element $x_{i} \in X(i=1,2, \ldots, n)$, where $m$ is a fuzzy measure, defined as follows.

Definition 6 (see [38]). A fuzzy measure $m$ on the set $X$ is a set function $m: \theta(x) \rightarrow[0,1]$ satisfying the following axioms:

(1) $m(\phi)=0, m(X)=1$;

(2) $A \subseteq B$ implies that $m(A) \leq m(B)$, for all $A, B \subseteq X$;

(3) $m(A \cup B)=m(A)+m(B)+\rho m(A) m(B)$, for all $A, B \subseteq$ $X$ and $A \cap B=\phi$, where $\rho \in(-1, \infty)$. 
Particularly, if $\rho=0$, then the condition (3) reduces to the axiom of additive measure:

$$
m(A \cup B)=m(A)+m(B), \quad \forall A, B \subseteq X, A \cap B=\phi .
$$

If all the elements in $X$ are independent, then we have

$$
m(A)=\sum_{x_{i} \in A} m\left(\left\{x_{i}\right\}\right), \quad \forall A \subseteq X .
$$

Based on Definition 5, in what follows we use the wellknown Choquet integral [29] to develop an operator for aggregating the interval grey uncertain linguistic variables with correlative weights.

Definition 7 . Let $m$ be a fuzzy measure on $X$, and Let $\underset{\otimes}{\widetilde{A}}\left(x_{j}\right)=\left(\left[s_{\alpha_{j}}, s_{\beta_{j}}\right],\left[g_{j}^{L}, g_{j}^{U}\right]\right)(j=1,2, \ldots, n)$ be $n$ interval grey uncertain linguistic variables, then we call

$$
\text { (C) } \begin{aligned}
& \int_{\otimes} \underset{\otimes}{\widetilde{A}}(x) d m \\
= & \operatorname{IGULCOA}\left(\underset{\otimes}{\widetilde{A}}\left(x_{1}\right), \underset{\otimes}{\widetilde{A}}\left(x_{2}\right), \ldots, \underset{\otimes}{\widetilde{A}}\left(x_{n}\right)\right) \\
= & \bigoplus_{j=1}^{n}\left(m\left(B\left(x_{\tau(j)}\right)\right)-m\left(B\left(x_{\tau(j-1)}\right)\right)\right) \underset{\otimes}{\widetilde{A}}\left(x_{\tau(j)}\right)
\end{aligned}
$$

the interval grey uncertain linguistic correlated ordered arithmetic averaging (IGULCOA) operator, where (C) $\int \underset{\otimes}{\widetilde{A}} d m$ denotes the Choquet integral, $(\tau(1), \tau(2), \ldots, \tau(n))$ is a permutation of $(1,2, \ldots, n)$ such that $\underset{\otimes}{\widetilde{A}}\left(x_{\tau(1)}\right) \geq \underset{\otimes}{\widetilde{A}\left(x_{\tau(2)}\right) \geq}$ $\cdots \geq \underset{\otimes}{\widetilde{A}}\left(x_{\tau(n)}\right), B\left(x_{\tau(j)}\right)=\left\{x_{\tau(k)} \mid k \leq j, k \geq 1\right\}$, and $B\left(x_{\tau(0)}\right)^{\otimes}=\phi$, whose aggregated value is also an interval grey uncertain linguistic variable ((IGULV), for short).

Below, we discuss two special cases of the IGULCOA operator.

(1) If $\rho=0$, then $m(B \cup C)=m(B)+m(C)$ and $m\left(\left\{x_{\tau(j)}\right\}\right)=$ $m\left(B\left(x_{\tau(j)}\right)\right)-m\left(B\left(x_{\tau(j-1)}\right)\right), j=1,2, \ldots, n$. In this case, the IGULCOA operator (9) reduces to the interval grey uncertain linguistic weighted arithmetic averaging (IGULWAA) operator:

$$
\begin{aligned}
& \operatorname{IGULWAA}\left(\underset{\otimes}{\widetilde{A}}\left(x_{1}\right), \underset{\otimes}{\widetilde{A}}\left(x_{2}\right), \ldots, \underset{\otimes}{\widetilde{A}}\left(x_{n}\right)\right) \\
& =\bigoplus_{j=1}^{n} m\left(\left\{x_{j}\right\}\right) \underset{\otimes}{\widetilde{A}}\left(x_{j}\right) \\
& =\left(\left[s_{\sum_{j=1}^{n} m\left(\left\{x_{j}\right\}\right) \alpha_{j}}, s_{\sum_{j=1}^{n} m\left(\left\{x_{j}\right\}\right) \beta_{j}}\right],\right. \\
& \\
& \left.\left[1-\prod_{j=1}^{n}\left(1-g_{j}^{L}\right), 1-\prod_{j=1}^{n}\left(1-g_{j}^{U}\right)\right]\right) .
\end{aligned}
$$

In particular, if $m\left(\left\{x_{j}\right\}\right)=1 / n$, for all $j=1,2, \ldots, n$, then the IGULWAA operator (10) reduces to the interval grey uncertain linguistic arithmetic averaging (IGULAA) operator:

$$
\begin{aligned}
\operatorname{IGULAA} & \left(\underset{\otimes}{\widetilde{A}}\left(x_{1}\right), \underset{\otimes}{\widetilde{A}}\left(x_{2}\right), \ldots, \underset{\otimes}{\widetilde{A}}\left(x_{n}\right)\right) \\
= & \left\{\left[s_{\sum_{j=1}^{n}\left(\alpha_{j} / n\right)}, s_{\sum_{j=1}^{n}\left(\beta_{j} / n\right)}\right],\right. \\
& {\left.\left[1-\prod_{j=1}^{n}\left(1-g_{j}^{L}\right), 1-\prod_{j=1}^{n}\left(1-g_{j}^{U}\right)\right]\right\}, }
\end{aligned}
$$

(2) If $m(B)=\sum_{j=1}^{|B|} \omega_{j}$, for all $B \subseteq X$, where $|B|$ is the number of the elements in the set $B$, then $\omega_{j}=m\left(B\left(x_{\tau(j)}\right)\right)-$ $m\left(B\left(x_{\tau(j-1)}\right)\right), j=1,2, \ldots, n$, where $\omega=\left(\omega_{1}, \omega_{2}, \ldots, \omega_{n}\right)^{T}$, $\omega_{j} \geq 0, j=1,2, \ldots, n$, and $\sum_{j=1}^{n} \omega_{j}=1$. In this case, the IGULCOA operator (9) reduces to the interval grey uncertain linguistic ordered weighted arithmetic averaging (IGULOWA) operator:

$$
\begin{aligned}
& \operatorname{IGULOWA}\left(\underset{\otimes}{\widetilde{A}}\left(x_{1}\right), \underset{\otimes}{\widetilde{A}}\left(x_{2}\right), \ldots, \underset{\otimes}{\widetilde{A}}\left(x_{n}\right)\right)=\bigoplus_{j=1}^{n} \omega_{j} \widetilde{A}\left(x_{\tau(j)}\right) \\
& =\left(\left[s_{\left.\sum_{j=1}^{n} \omega_{j} \alpha_{\tau(j)}, s_{\sum_{j=1}^{n} \omega_{j} \beta_{\tau(j)}}\right],}\right.\right. \\
& \left.\left.\qquad 1-\prod_{j=1}^{n}\left(1-g_{\tau(j)}^{L}\right), 1-\prod_{j=1}^{n}\left(1-g_{\tau(j)}^{U}\right)\right]\right) .
\end{aligned}
$$

In particular, if $m(B)=|B| / n$ for all $B \subseteq X$, then both the IGULCOA operator (9) and the IGULOWA operator (12) reduce to the IGULAA operator.

Definition 8. Let $\underset{\otimes}{\widetilde{A}}\left(x_{j}\right)=\left(\left[s_{\alpha_{j}}, s_{\beta_{j}}\right],\left[g_{j}^{L}, g_{j}^{U}\right]\right)(j=1,2, \ldots, n)$ be a collection of interval grey uncertain linguistic variables, and, $\mu$ be a fuzzy measure on $X$, an induced interval grey uncertain linguistic correlated ordered arithmetic averaging (I-IGULCOA) operator is defined as follows:

$$
\begin{aligned}
& \operatorname{I-IGLCOA}\left(\left\langle u_{1}, \underset{\otimes}{\widetilde{A}}\left(x_{1}\right)\right\rangle,\left\langle u_{2}, \underset{\otimes}{\widetilde{A}}\left(x_{2}\right)\right\rangle, \ldots,\left\langle u_{n}, \underset{\otimes}{\widetilde{A}}\left(x_{n}\right)\right\rangle\right) \\
& =\bigoplus_{j=1}^{n}\left(m\left(B\left(x_{\sigma(j)}\right)\right)-m\left(B\left(x_{\sigma(j-1)}\right)\right)\right) \underset{\otimes}{\widetilde{A}}\left(x_{\sigma(j)}\right) \\
& =\left(\left[s_{\sum_{j=1}^{n}\left(m\left(B\left(x_{\sigma(j)}\right)\right)-m\left(B\left(x_{\sigma(j-1)}\right)\right)\right) \alpha_{\sigma(j)},}\right.\right. \\
& \left.\quad s_{\sum_{j=1}^{n}\left(m\left(B\left(x_{\sigma(j)}\right)\right)-m\left(B\left(x_{\sigma(j-1)}\right)\right)\right) \beta_{\sigma(j)}}\right], \\
& \left.\quad\left[1-\prod_{j=1}^{n}\left(1-g_{\sigma(j)}^{L}\right), 1-\prod_{j=1}^{n}\left(1-g_{\sigma(j)}^{U}\right)\right]\right),
\end{aligned}
$$


where $(\sigma(1), \sigma(2), \ldots, \sigma(n))$ is a permutation of $(1,2, \ldots, n)$, for all $j=1,2, \ldots, n$; that is, $\left\langle u_{j}, \widetilde{A}\left(x_{j}\right)\right\rangle$ is the 2-tuple with $u_{\sigma(j)}$ the $j$ th largest values in the set $\left\{u_{1}, u_{2}, \ldots, u_{n}\right\}$, and $u_{j}$ in $\left\langle u_{j}, \underset{\otimes}{\widetilde{A}}\left(x_{j}\right)\right\rangle$ is referred to as the order-inducing variable and $\underset{\otimes}{\widetilde{A}}\left(x_{j}\right)=\left(\left[s_{\alpha_{j}}, s_{\beta_{j}}\right],\left[g_{j}^{L}, g_{j}^{U}\right]\right)$ as the interval grey uncertain linguistic value, $B\left(x_{\sigma(j)}\right)=\left\{x_{\sigma(k)} \mid k \leq j, k \geq 1\right\}$, for $k \geq 1$, and $B\left(x_{\sigma(0)}\right)=\phi$.

In the above definition, the reordering of the set of values to aggregate $\left(\widetilde{A}\left(x_{1}\right), \widetilde{A}\left(x_{2}\right), \ldots, \widetilde{A}\left(x_{n}\right)\right)$ is induced by the reordering of the set of values $\left\{u_{1}, u_{2}, \ldots, u_{n}\right\}$ associated with them, which is based on their magnitude. The main difference between the IGULCOA operator and the I-IGULCOA operator resides in the reordering step of the argument variable. In the case of IGULCOA operator, this reordering is based on the magnitude of the interval grey uncertain linguistic values to be aggregated, whereas in the case of I-IGULCOA operator an order-inducing variable is used as the criterion to induce that reordering. Obviously, in Definition 8, if $u_{j}=\widetilde{A}\left(x_{j}\right)$, for all $j$, then the I-IGULCOA operator is reduced to the IGULCOA operator.

Similar to the other induced operators, the I-IGULCOA operator has the following properties.

Theorem 9 (commutativity). Consider

$$
\begin{gathered}
\operatorname{I-IGULCOA}\left(\left\langle u_{1}, \underset{\otimes}{\widetilde{A}}\left(x_{1}\right)\right\rangle,\left\langle u_{2}, \underset{\otimes}{\widetilde{A}}\left(x_{2}\right)\right\rangle, \ldots,\left\langle u_{n}, \underset{\otimes}{\widetilde{A}}\left(x_{n}\right)\right\rangle\right) \\
=\operatorname{I-IGULCOA}\left(\left\langle u_{1}^{\prime}, \widetilde{A}_{\otimes}^{\prime}\left(x_{1}\right)\right\rangle,\left\langle u_{2}^{\prime}, \widetilde{A}^{\prime}\left(x_{2}\right)\right\rangle, \ldots,\right. \\
\left.\left\langle u_{n}^{\prime}, \widetilde{A}_{\otimes}^{\prime}\left(x_{n}\right)\right\rangle\right),
\end{gathered}
$$

where $\left(\left\langle u_{1}^{\prime}, \widetilde{A}_{\otimes}^{\prime}\left(x_{1}\right)\right\rangle,\left\langle u_{2}^{\prime}, \widetilde{A}_{\otimes}^{\prime}\left(x_{2}\right)\right\rangle, \ldots,\left\langle u_{n}^{\prime}, \widetilde{A}_{\otimes}^{\prime}\left(x_{n}\right)\right\rangle\right)$ is any permutation of $\left(\left\langle u_{1}, \underset{\otimes}{\widetilde{A}}\left(x_{1}\right)\right\rangle,\left\langle u_{2}, \underset{\otimes}{\widetilde{A}}\left(x_{2}\right)\right\rangle, \ldots,\left\langle u_{n}, \underset{\otimes}{\widetilde{A}}\left(x_{n}\right)\right\rangle\right)$.

Proof. Let

$\operatorname{I-IGULCOA}\left(\left\langle u_{1}, \underset{\otimes}{\widetilde{A}}\left(x_{1}\right)\right\rangle,\left\langle u_{2}, \underset{\otimes}{\widetilde{A}}\left(x_{2}\right)\right\rangle, \ldots,\left\langle u_{n}, \widetilde{A}\left(x_{n}\right)\right\rangle\right)$

$=\bigoplus_{j=1}^{n}\left(m\left(B\left(x_{\sigma(j)}\right)\right)-m\left(B\left(x_{\sigma(j-1)}\right)\right)\right) \underset{\otimes}{\widetilde{A}}\left(x_{\sigma(j)}\right)$

$\operatorname{I-IGULCOA}\left(\left\langle u_{1}^{\prime}, \underset{\otimes}{\widetilde{A}^{\prime}}\left(x_{1}\right)\right\rangle,\left\langle u_{2}^{\prime}, \underset{\otimes}{\widetilde{A}^{\prime}}\left(x_{2}\right)\right\rangle, \ldots\right.$,

$$
\left.\left\langle u_{n}^{\prime}, \widetilde{A}_{\otimes}^{\prime}\left(x_{n}\right)\right\rangle\right)
$$

$=\bigoplus_{j=1}^{n}\left(m\left(B\left(x_{\sigma(j)}\right)\right)-m\left(B\left(x_{\sigma(j-1)}\right)\right)\right) \underset{\otimes}{\widetilde{A}^{\prime}}\left(x_{\sigma(j)}\right)$.
Since $\left(\left\langle u_{1}^{\prime}, \widetilde{A}_{\otimes}^{\prime}\left(x_{1}\right)\right\rangle,\left\langle u_{2}^{\prime}, \widetilde{A}_{\otimes}^{\prime}\left(x_{2}\right)\right\rangle, \ldots,\left\langle u_{n}^{\prime}, \widetilde{A}_{\otimes}^{\prime}\left(x_{n}\right)\right\rangle\right)$ is any permutation of $\left.\left(\left\langle u_{1}, \widetilde{A}\left(x_{1}\right)\right\rangle,\left\langle u_{2}, \underset{\otimes}{\widetilde{A}}\left(x_{2}\right)\right\rangle, \ldots,\left\langle u_{n}, \underset{\otimes}{\widetilde{A}\left(x_{n}\right.}\right)\right\rangle\right)$, we have $\underset{\otimes}{\mathbb{A}}\left(x_{j}\right)=\underset{\otimes}{\widetilde{A}^{\prime}}\left(x_{j}\right)(j=1,2, \ldots, n)$, and then

$$
\begin{aligned}
& \operatorname{I-IGULCOA}\left(\left\langle u_{1}, \underset{\otimes}{\widetilde{A}}\left(x_{1}\right)\right\rangle,\left\langle u_{2}, \underset{\otimes}{\widetilde{A}}\left(x_{2}\right)\right\rangle, \ldots,\left\langle u_{n}, \underset{\otimes}{\widetilde{A}}\left(x_{n}\right)\right\rangle\right) \\
& =\operatorname{I-IGULCOA}\left(\left\langle u_{1}^{\prime}, \underset{\otimes}{\widetilde{A}^{\prime}}\left(x_{1}\right)\right\rangle,\left\langle u_{2}^{\prime}, \underset{\otimes}{\widetilde{A}^{\prime}\left(x_{2}\right)}\right\rangle, \ldots,\right. \\
& \left.\left\langle u_{n}^{\prime}, \widetilde{A}_{\otimes}^{\prime}\left(x_{n}\right)\right\rangle\right) \text {. }
\end{aligned}
$$

Theorem 10 (idempotency). If $\underset{\otimes}{\widetilde{A}}\left(x_{j}\right)=\left(\left[s_{\alpha_{j}}, s_{\beta_{j}}\right],\left[g_{j}^{L}, g_{j}^{U}\right]\right)=$ $\widetilde{\otimes}(x)=\left(\left[s_{\alpha}, s_{\beta}\right],\left[g^{L}, g^{U}\right]\right)(j=1,2, \ldots, n)$, for all $j$, then

$$
\begin{gathered}
\operatorname{I-IGULCOA}\left(\left\langle u_{1}, \underset{\otimes}{\widetilde{A}}\left(x_{1}\right)\right\rangle,\left\langle u_{2}, \underset{\otimes}{\widetilde{A}}\left(x_{2}\right)\right\rangle, \ldots,\left\langle u_{n}, \underset{\otimes}{\widetilde{A}}\left(x_{n}\right)\right\rangle\right) \\
=\underset{\otimes}{\widetilde{A}}(x)=\left(\left[s_{\alpha}, s_{\beta}\right],\left[g^{L}, g^{U}\right]\right) .
\end{gathered}
$$

Proof. Since $\underset{\otimes}{\widetilde{A}}\left(x_{j}\right)=\underset{\otimes}{\widetilde{A}}(x)$, for all $j$, we have

$$
\begin{aligned}
& \text { I-IGULCOA }\left(\left\langle u_{1}, \underset{\otimes}{\widetilde{A}(}\left(x_{1}\right)\right\rangle,\left\langle u_{2}, \underset{\otimes}{\widetilde{A}}\left(x_{2}\right)\right\rangle, \ldots,\left\langle u_{n}, \underset{\otimes}{\widetilde{A}}\left(x_{n}\right)\right\rangle\right) \\
& =\bigoplus_{j=1}^{n}\left(m\left(B\left(x_{\sigma(j)}\right)\right)-m\left(B\left(x_{\sigma(j-1)}\right)\right)\right) \underset{\otimes}{\widetilde{A}}\left(x_{\sigma(j)}\right) \\
& =\bigoplus_{j=1}^{n}\left(m\left(B\left(x_{\sigma(j)}\right)\right)-m\left(B\left(x_{\sigma(j-1)}\right)\right)\right) \underset{\otimes}{\widetilde{A}}(x) \\
& =\underset{\otimes}{\widetilde{A}}(x) .
\end{aligned}
$$

Theorem 11 (monotonicity). If $\underset{\otimes}{\widetilde{A}}\left(x_{j}\right) \leq \underset{\otimes}{\widetilde{A}^{\prime}}\left(x_{j}\right)(j=1,2$, $\ldots, n)$, then

$$
\begin{gathered}
\operatorname{I-IGULCOA}\left(\left\langle u_{1}, \underset{\otimes}{\widetilde{A}}\left(x_{1}\right)\right\rangle,\left\langle u_{2}, \underset{\otimes}{\widetilde{A}}\left(x_{2}\right)\right\rangle, \ldots,\left\langle u_{n}, \underset{\otimes}{\widetilde{A}}\left(x_{n}\right)\right\rangle\right) \\
\leq \operatorname{I-IGULCOA}\left(\left\langle u_{1}^{\prime}, \widetilde{\otimes}_{\otimes}^{\widetilde{A}^{\prime}}\left(x_{1}\right)\right\rangle,\left\langle u_{2}^{\prime}, \widetilde{\otimes}_{\otimes}^{\widetilde{A}^{\prime}}\left(x_{2}\right)\right\rangle, \ldots,\right. \\
\left.\left\langle u_{n}^{\prime}, \underset{\otimes}{\widetilde{A}^{\prime}}\left(x_{n}\right)\right\rangle\right) .
\end{gathered}
$$


Proof. Let

$$
\begin{aligned}
& \operatorname{I-IGULCOA}\left(\left\langle u_{1}, \underset{\otimes}{\widetilde{A}}\left(x_{1}\right)\right\rangle,\left\langle u_{2}, \underset{\otimes}{\widetilde{A}}\left(x_{2}\right)\right\rangle, \ldots,\left\langle u_{n}, \underset{\otimes}{\widetilde{A}}\left(x_{n}\right)\right\rangle\right) \\
& =\bigoplus_{j=1}^{n}\left(m\left(B\left(x_{\sigma(j)}\right)\right)-m\left(B\left(x_{\sigma(j-1)}\right)\right)\right) \underset{\otimes}{\widetilde{A}}\left(x_{\sigma(j)}\right)
\end{aligned}
$$$$
\operatorname{I-IGULCOA}\left(\left\langle u_{1}, \underset{\otimes}{\widetilde{A}^{\prime}}\left(x_{1}\right)\right\rangle,\left\langle u_{2}, \widetilde{A}_{\otimes}^{\prime}\left(x_{2}\right)\right\rangle, \ldots,\right.
$$$$
\left.\left\langle u_{n}, \widetilde{A}_{\otimes}^{\prime}\left(x_{n}\right)\right\rangle\right)
$$$$
=\bigoplus_{j=1}^{n}\left(m\left(B\left(x_{\sigma(j)}\right)\right)-m\left(B\left(x_{\sigma(j-1)}\right)\right)\right) \underset{\otimes}{\widetilde{A}^{\prime}}\left(x_{\sigma(j)}\right) .
$$

Since $\underset{\otimes}{\widetilde{A}}\left(x_{j}\right) \leq \underset{\otimes}{\widetilde{A}^{\prime}}\left(x_{j}\right)(j=1,2, \ldots, n)$, it follows that $\underset{\otimes}{\widetilde{A}}\left(x_{\sigma(j)}\right) \leq \underset{\otimes}{\widetilde{A}^{\prime}}\left(x_{\sigma(j)}\right)(j=1,2, \ldots, n)$, then

$\operatorname{I-IGULCOA}\left(\left\langle u_{1}, \underset{\otimes}{\widetilde{A}}\left(x_{1}\right)\right\rangle,\left\langle u_{2}, \underset{\otimes}{\widetilde{A}}\left(x_{2}\right)\right\rangle, \ldots,\left\langle u_{n}, \underset{\otimes}{\widetilde{A}}\left(x_{n}\right)\right\rangle\right)$

$$
\leq \operatorname{I-IGULCOA}\left(\left\langle u_{1}^{\prime}, \widetilde{A}_{\otimes}^{\prime}\left(x_{1}\right)\right\rangle,\left\langle u_{2}^{\prime}, \widetilde{A}_{\otimes}^{\prime}\left(x_{2}\right)\right\rangle, \ldots,\right.
$$

$$
\left.\left\langle u_{n}^{\prime}, \widetilde{A}_{\otimes}^{\prime}\left(x_{n}\right)\right\rangle\right) .
$$

Theorem 12 (boundness). Consider

$$
\begin{aligned}
& \underset{j}{\operatorname{Min}} \underset{\otimes}{\widetilde{A}}\left(x_{j}\right) \\
& \leq \operatorname{I-IGULCOA}\left(\left\langle u_{1}, \underset{\otimes}{\widetilde{A}}\left(x_{1}\right)\right\rangle,\left\langle u_{2}, \underset{\otimes}{\widetilde{A}}\left(x_{2}\right)\right\rangle, \ldots,\right. \\
& \left.\qquad\left\langle u_{n}, \underset{\otimes}{\widetilde{A}}\left(x_{n}\right)\right\rangle\right) \\
& \leq \operatorname{Max}_{j} \underset{\otimes}{\widetilde{A}}\left(x_{j}\right) .
\end{aligned}
$$

Proof. Let

$$
\begin{gathered}
\operatorname{I-IGULCOA}\left(\left\langle u_{1}, \underset{\otimes}{\widetilde{A}}\left(x_{1}\right)\right\rangle,\left\langle u_{2}, \underset{\otimes}{\widetilde{A}}\left(x_{2}\right)\right\rangle, \ldots,\left\langle u_{n}, \underset{\otimes}{\widetilde{A}}\left(x_{n}\right)\right\rangle\right) \\
=\bigoplus_{j=1}^{n}\left(m\left(B\left(x_{\sigma(j)}\right)\right)-m\left(B\left(x_{\sigma(j-1)}\right)\right)\right) \underset{\otimes}{\widetilde{A}}\left(x_{\sigma(j)}\right) .
\end{gathered}
$$

Since $\operatorname{Min}_{j} \underset{\otimes}{\widetilde{A}}\left(x_{j}\right) \leq \underset{\otimes}{\widetilde{A}}\left(x_{j}\right) \leq \operatorname{Max}_{j} \underset{\otimes}{\widetilde{A}}\left(x_{j}\right)(j=1,2, \ldots, n)$, then

$$
\begin{aligned}
& \bigoplus_{j=1}^{n}\left(m\left(B\left(x_{\sigma(j)}\right)\right)-m\left(B\left(x_{\sigma(j-1)}\right)\right)\right) \operatorname{Min}_{j} \underset{\otimes}{\widetilde{A}}\left(x_{j}\right) \\
& \leq \bigoplus_{j=1}^{n}\left(m\left(B\left(x_{\sigma(j)}\right)\right)-m\left(B\left(x_{\sigma(j-1)}\right)\right)\right) \underset{\otimes}{\widetilde{A}}\left(x_{\sigma(j)}\right) \\
& \leq \bigoplus_{j=1}^{n}\left(m\left(B\left(x_{\sigma(j)}\right)\right)-m\left(B\left(x_{\sigma(j-1)}\right)\right)\right) \underset{j}{\operatorname{Max}} \underset{\otimes}{\widetilde{A}}\left(x_{j}\right)
\end{aligned}
$$$$
\bigoplus_{j=1}^{n}\left(m\left(B\left(x_{\sigma(j)}\right)\right)-m\left(B\left(x_{\sigma(j-1)}\right)\right)\right) \operatorname{Min}_{j} \underset{\otimes}{\widetilde{A}}\left(x_{j}\right)
$$$$
=\operatorname{Min}_{j} \underset{\otimes}{\widetilde{A}}\left(x_{j}\right)
$$$$
\bigoplus_{j=1}^{n}\left(m\left(B\left(x_{\sigma(j)}\right)\right)-m\left(B\left(x_{\sigma(j-1)}\right)\right)\right) \operatorname{Max}_{j} \underset{\otimes}{\widetilde{A}}\left(x_{j}\right)
$$$$
=\operatorname{Max}_{j} \underset{\otimes}{\widetilde{A}}\left(x_{j}\right) .
$$

Hence,

$$
\begin{aligned}
& \underset{j}{\operatorname{Min}} \underset{\otimes}{\widetilde{A}}\left(x_{j}\right) \\
& \leq \operatorname{I-IGULCOA}\left(\left\langle u_{1}, \underset{\otimes}{\widetilde{A}}\left(x_{1}\right)\right\rangle,\left\langle u_{2}, \underset{\otimes}{\widetilde{A}}\left(x_{2}\right)\right\rangle, \ldots,\right. \\
& \left.\qquad\left\langle u_{n}, \underset{\otimes}{\widetilde{A}}\left(x_{n}\right)\right\rangle\right) \\
& \leq \operatorname{Max}_{j} \underset{\otimes}{\widetilde{A}}\left(x_{j}\right) .
\end{aligned}
$$

\section{An Approach to Multiple Attribute Group Decision Making Method Based on the IGULCOA Operator and the I-IGULCOA Operator}

In this section, we will develop an approach to multiple attribute group decision making with interval grey uncertain linguistic variables information and correlated weight as follows.

Let $A=\left\{a_{1}, a_{2}, \ldots, a_{m}\right\}$ be a discrete set of alternatives, and let $X=\left\{x_{1}, x_{2}, \ldots, x_{n}\right\}$ be the set of attributes, $m$ a fuzzy measure on $X$, where $0 \leq m\left(\left\{x_{1}, \ldots, x_{j}\right\}\right) \leq 1, m(\{X\})=$ 1 and $m(\{\phi\})=0$. Let $D=\left\{d_{1}, d_{2}, \ldots, d_{t}\right\}$ be the set of decision makers, $\mu$ a fuzzy measure on $D$, where $0 \leq$ $\mu\left(\left\{d_{1}, \ldots, d_{k}\right\}\right) \leq 1, \mu(\{D\})=1$, and $\mu(\{\phi\})=0$. Suppose that $\widetilde{R}_{k}=\left(\underset{\otimes_{i}}{\widetilde{A}^{(k)}}\left(x_{j}\right)\right)_{m \times n}$ is the interval grey uncertain linguistic decision matrix, where $\widetilde{\otimes}_{\otimes_{i}}^{(k)}\left(x_{j}\right)$ indicates the attribute value that the alternative $a_{i}$ satisfies the attribute $x_{j}$ given by the 
decision maker $d_{k}$, and $u_{j}^{(k)}$ indicates the inducing values that the decision maker $d_{k}$ satisfies the attribute $x_{j}, i=$ $1,2, \ldots, m, j=1,2, \ldots, n$, and $k=1,2, \ldots, t$.

In the following, we apply the IGULCOA and IIGULCOA operators to multiple attribute group decision making based on interval grey uncertain linguistic information and correlated weight. The method involves the following steps.

Step 1. Utilize the decision information given in matrix $\widetilde{R}_{k}$, and the I-IGULCOA operator which has correlative weights vector:

$$
\begin{aligned}
& r_{i}^{(k)} \\
& =\operatorname{I-IGLCOA}\left(\left\langle u_{1}, \widetilde{A}_{\otimes_{i}}^{(k)}\left(x_{1}\right)\right\rangle,\left\langle u_{2},{\widetilde{\otimes_{i}}}^{(k)}\left(x_{2}\right)\right\rangle, \ldots,\right. \\
& \left.\quad\left(\left\langle u_{n}, \widetilde{A}_{\otimes_{i}}^{(k)}\left(x_{n}\right)\right\rangle\right)\right) \\
& \left.=\bigoplus_{j=1}^{n}\left(m\left(B\left(x_{\sigma(j)}\right)\right)-m\left(B\left(x_{\sigma(j-1)}\right)\right)\right)\right)_{\otimes_{i}}^{\widetilde{A}^{(k)}}\left(x_{\sigma(j)}\right) \\
& =\left(\left[s_{\sum_{j=1}^{n}\left(m\left(B\left(x_{\sigma(j)}\right)\right)-m\left(B\left(x_{\sigma(j-1)}\right)\right)\right) \alpha_{\sigma(j)},}\right.\right. \\
& s_{\left.\sum_{j=1}^{n}\left(m\left(B\left(x_{\sigma(j)}\right)\right)-m\left(B\left(x_{\sigma(j-1)}\right)\right)\right) \beta_{\sigma(j)}\right],} \\
& {\left[\begin{array}{c}
\left.\left.1-\prod_{j=1}^{n}\left(1-g_{\sigma(j)}^{L}\right), 1-\prod_{j=1}^{n}\left(1-g_{\sigma(j)}^{U}\right)\right]\right) \\
i=1,2, \ldots, m, j=1,2, \ldots, n, k=1,2, \ldots, t
\end{array}\right.}
\end{aligned}
$$

to aggregate all the decision matrices $\widetilde{R}_{k}(k=1,2, \ldots, t)$ into a collective decision matrix $\widetilde{R}=\left(r_{i}^{(k)}\right)_{m \times t}$, where $m$ is a fuzzy measure on $X, 0 \leq m\left(\left\{x_{1}, \ldots, x_{j}\right\}\right) \leq 1, m(\{X\})=1$, and $m(\{\phi\})=0(j=1,2, \ldots, n)$.

Step 2. Utilize the decision information given in matrix $\widetilde{R}$ and the IGULCOA operator:

$$
\begin{array}{r}
\widetilde{z}_{i}=\operatorname{IGULCOA}\left(\widetilde{r}_{i}^{(1)}, \tilde{r}_{i}^{(2)}, \ldots, \widetilde{r}_{i}^{(t)}\right) \\
=\bigoplus_{k=1}^{t}\left(\mu\left(B\left(d_{\tau(k)}\right)\right)-\mu\left(B\left(d_{\tau(k-1)}\right)\right)\right) \tilde{r}_{i}^{(k)} \\
i=1,2, \ldots, m
\end{array}
$$

to derive the collective overall preference values $\widetilde{r}_{i}(i=$ $1,2, \ldots, m)$ of the alternative $A_{i}$, where $\mu$ is a fuzzy measure on $D, 0 \leq \mu\left(\left\{d_{1}, \ldots, d_{k}\right\}\right) \leq 1, \mu(\{D\})=1$, and $\mu(\{\phi\})=0$.

Step 3. Let the basic unit-interval monotonic (BUM) function be $\rho(y)=y^{\delta}$. We get the size of the collective overall pre-ference values $\widetilde{z}_{i}(i=1,2, \ldots, m)$ by using

$$
\begin{aligned}
\widetilde{Q}\left(\widetilde{z}_{i}\right) & \\
= & {\left[s_{\alpha_{i}}, s_{\beta_{i}}\right] \times f_{\rho}\left(\left[\left(1-g_{i}^{U}\right),\left(1-g_{i}^{L}\right)\right]\right) } \\
& =\left[s_{\alpha_{i}}, s_{\beta_{i}}\right] \times \int_{0}^{1} \frac{d \rho(y)}{d y}\left[\left(1-g_{i}^{L}\right)-y\left(g_{i}^{U}-g_{i}^{L}\right)\right] d y .
\end{aligned}
$$

Step 4. To rank these collective overall preference values $\widetilde{Q}\left(\widetilde{z}_{i}\right)(i=1,2, \ldots, m)$, we first compare each $\widetilde{Q}\left(\widetilde{z}_{i}\right)$ with all $\widetilde{\mathrm{Q}}\left(\widetilde{z}_{i}\right)(i=1,2, \ldots, m)$ by using $(9)$. For simplicity, we let $p_{i j}=p\left(\widetilde{Q}\left(\widetilde{z}_{i}\right) \geq \widetilde{\mathrm{Q}}\left(\widetilde{z}_{j}\right)\right)$, then we develop a complementary matrix as $P=\left(p_{i j}\right)_{n \times n}$, where $p_{i j} \geq 0, p_{i j}+p_{j i}=1, p_{i i}=1 / 2$, $i, j=1,2, \ldots, n$. Summing all elements in each line of matrix $P$, we have $p_{i}=\sum_{j=1}^{n} p_{i j}, i=1,2, \ldots, n$. Consider

$$
\begin{aligned}
p\left(\widetilde{Q}\left(\widetilde{z}_{i}\right) \geq \widetilde{Q}\left(\widetilde{z}_{j}\right)\right) \\
=\frac{\operatorname{Max}\left\{0, \operatorname{len}\left(\widetilde{s}_{i}\right)+\operatorname{len}\left(\widetilde{s}_{j}\right)\right\}-\operatorname{Max}\left(\beta_{j}-\alpha_{i}, 0\right)}{\operatorname{len}\left(\widetilde{s}_{i}\right)+\operatorname{len}\left(\widetilde{s}_{j}\right)} .
\end{aligned}
$$

Then, we rank $\widetilde{Q}\left(\widetilde{z}_{i}\right)(i=1,2, \ldots, m)$ in a descending order in accordance with the values of $p_{i}(i=1,2, \ldots, m)$.

Step 5. The ranking of the alternatives can be gained and the best one can be found out.

\section{Illustrative Example}

Let us suppose there is an investment company, which wants to invest a sum of money in the best option. There is a panel with four possible alternatives to invest the money:

(1) $a_{1}$ is a car company.

(2) $a_{2}$ is a food company.

(3) $a_{3}$ is a computer company.

(4) $a_{4}$ is an arms company.

The investment company must take a decision according to the following four attributes:

(1) $x_{1}$ is the risk analysis.

(2) $x_{2}$ is the growth analysis.

(3) $x_{3}$ is the social-political impact analysis.

(4) $x_{4}$ is the environmental impact analysis.

The four possible alternatives $\left\{a_{1}, a_{2}, a_{3}, a_{4}\right\}$ are to be evaluated by the three decision makers $\left\{d_{1}, d_{2}, d_{3}\right\}$ under the above four attributes and construct, respectively, the inducing 
TABLE 1: Inducing variables $U$.

\begin{tabular}{lcccc}
\hline Experts & $\begin{array}{c}\text { Attribute } \\
\left(x_{1}\right)\end{array}$ & $\begin{array}{c}\text { Attribute } \\
\left(x_{2}\right)\end{array}$ & $\begin{array}{c}\text { Attribute } \\
\left(x_{3}\right)\end{array}$ & $\begin{array}{c}\text { Attribute } \\
\left(x_{4}\right)\end{array}$ \\
\hline$D_{1}$ & 17 & 15 & 22 & 12 \\
$D_{2}$ & 15 & 22 & 25 & 13 \\
$D_{3}$ & 16 & 21 & 25 & 28 \\
\hline
\end{tabular}

variables $U$, which are shown in Table 1 . And the decision matrices $\widetilde{R}_{k}=\underset{\otimes_{i}}{\left(\widetilde{A}^{(k)}\left(x_{j}\right)\right)}$ 4×4 are as follows:

$$
\begin{aligned}
& \widetilde{R}_{1}=\left[\begin{array}{ll}
\left(\left[s_{4}, s_{5}\right],[0.2,0.5]\right) & \left(\left[s_{3}, s_{4}\right],[0.3,0.4]\right) \\
\left(\left[s_{3}, s_{5}\right],[0.4,0.5]\right) & \left(\left[s_{4}, s_{5}\right],[0.4,0.5]\right) \\
\left(\left[s_{3}, s_{6}\right],[0.2,0.4]\right) & \left(\left[s_{3}, s_{4}\right],[0.2,0.4]\right) \\
\left(\left[s_{4}, s_{6}\right],[0.3,0.6]\right) & \left(\left[s_{2}, s_{5}\right],[0.2,0.5]\right)
\end{array}\right. \\
& \left.\left(\left[s_{4}, s_{5}\right],[0.3,0.5]\right)\left(\left[s_{2}, s_{5}\right],[0.2,0.4]\right)\right] \\
& \left(\left[s_{2}, s_{4}\right],[0.2,0.4]\right)\left(\left[s_{3}, s_{4}\right],[0.3,0.5]\right) \\
& \left(\left[s_{3}, s_{4}\right],[0.3,0.4]\right)\left(\left[s_{4}, s_{5}\right],[0.2,0.5]\right) \\
& \left.\left(\left[s_{2}, s_{4}\right],[0.2,0.5]\right)\left(\left[s_{2}, s_{4}\right],[0.3,0.4]\right)\right] \\
& \widetilde{R}_{2}=\left[\begin{array}{ll}
\left(\left[s_{3}, s_{5}\right],[0.1,0.3]\right) & \left(\left[s_{2}, s_{4}\right],[0.2,0.3]\right) \\
\left(\left[s_{4}, s_{5}\right],[0.3,0.5]\right) & \left(\left[s_{2}, s_{5}\right],[0.3,0.4]\right) \\
\left(\left[s_{3}, s_{4}\right],[0.2,0.4]\right) & \left(\left[s_{3}, s_{4}\right],[0.2,0.3]\right) \\
\left(\left[s_{4}, s_{5}\right],[0.3,0.4]\right) & \left(\left[s_{3}, s_{5}\right],[0.4,0.5]\right)
\end{array}\right. \\
& \left(\left[s_{2}, s_{3}\right],[0.2,0.3]\right)\left(\left[s_{3}, s_{6}\right],[0.4,0.5]\right) \\
& \left(\left[s_{3}, s_{4}\right],[0.2,0.4]\right)\left(\left[s_{2}, s_{3}\right],[0.2,0.4]\right) \\
& \left(\left[s_{1}, s_{3}\right],[0.3,0.4]\right)\left(\left[s_{2}, s_{3}\right],[0.3,0.4]\right) \\
& \left.\left(\left[s_{1}, s_{4}\right],[0.3,0.5]\right)\left(\left[s_{3}, s_{4}\right],[0.2,0.5]\right)\right] \\
& \widetilde{R}_{3}=\left[\begin{array}{lc}
\left(\left[s_{4}, s_{5}\right],[0.2,0.4]\right) & \left(\left[s_{2}, s_{4}\right],[0.3,0.3]\right) \\
\left(\left[s_{3}, s_{4}\right],[0.3,0.4]\right) & \left(\left[s_{4}, s_{5}\right],[0.3,0.4]\right) \\
\left(\left[s_{3}, s_{4}\right],[0.2,0.3]\right) & \left(\left[s_{3}, s_{5}\right],[0.3,0.4]\right) \\
\left(\left[s_{2}, s_{5}\right],[0.2,0.3]\right) & \left(\left[s_{2}, s_{5}\right],[0.1,0.3]\right)
\end{array}\right. \\
& \left.\left(\left[s_{3}, s_{4}\right],[0.4,0.5]\right)\left(\left[s_{3}, s_{4}\right],[0.2,0.3]\right)\right] \\
& \left(\left[s_{1}, s_{4}\right],[0.1,0.4]\right)\left(\left[s_{2}, s_{5}\right],[0.1,0.3]\right) \\
& \left(\left[s_{1}, s_{3}\right],[0.1,0.5]\right)\left(\left[s_{3}, s_{4}\right],[0.2,0.3]\right) \\
& \left.\left(\left[s_{3}, s_{4}\right],[0.3,0.4]\right)\left(\left[s_{4}, s_{5}\right],[0.1,0.4]\right)\right]
\end{aligned}
$$

The experts evaluate the enterprises $a_{i}(i=1,2,3,4)$ in relation to the factors $x_{j}(j=1,2,3,4)$. Let

$$
\begin{array}{cl}
m\left(x_{1}\right)=0.3, & m\left(x_{2}\right)=0.35, \\
m\left(x_{3}\right)=0.30, & m\left(x_{4}\right)=0.22, \\
m\left(x_{1}, x_{2}\right)=0.7, & m\left(x_{1}, x_{3}\right)=0.6, \\
m\left(x_{1}, x_{4}\right)=0.55, & m\left(x_{2}, x_{3}\right)=0.5, \\
m\left(x_{2}, x_{4}\right)=0.45, & m\left(x_{3}, x_{4}\right)=0.4, \\
m\left(x_{1}, x_{2}, x_{3}\right)=0.82, & m\left(x_{1}, x_{2}, x_{4}\right)=0.87, \\
m\left(x_{1}, x_{3}, x_{4}\right)=0.75, & m\left(x_{2}, x_{3}, x_{4}\right)=0.6, \\
m\left(x_{1}, x_{2}, x_{3}, x_{4}\right)=1 .
\end{array}
$$

The fuzzy measure of weighting vector of decision makers $D_{k}(k=1,2,3)$ and sets of decision makes $D$ as follows:

$$
\begin{array}{rlrl}
\mu(\phi) & =0, & \mu\left(D_{1}\right)=0.25, \\
\mu\left(D_{2}\right) & =0.35, & \mu\left(D_{3}\right)=0.3, \\
\mu\left(D_{1}, D_{2}\right) & =0.7, & \mu\left(D_{1}, D_{3}\right)=0.65, \\
\mu\left(D_{2}, D_{3}\right) & =0.5, \quad \mu\left(D_{1}, D_{2}, D_{3}\right)=1 .
\end{array}
$$

Step 1. Utilize the decision information given in matrix $\widetilde{R}_{k}(k=1,2,3)$ inducing variables $U$, and the I-IGULCOA operator which has correlative weights vector:

$$
\begin{array}{r}
r_{i}^{(k)=} \operatorname{I-IGULCOA}\left(\left\langle u_{1},{\widetilde{\otimes_{i}}}^{\widetilde{A}^{(k)}}\left(x_{1}\right)\right\rangle,\left\langle u_{2}, \underset{\otimes_{i}}{\widetilde{A}^{(k)}}\left(x_{2}\right)\right\rangle,\right. \\
\left.\left\langle u_{3}, \underset{\otimes_{i}}{\widetilde{A}^{(k)}}\left(x_{3}\right)\right\rangle,\left\langle u_{4}, \underset{\otimes_{i}}{\widetilde{A}^{(k)}}\left(x_{4}\right)\right\rangle\right) \\
=\bigoplus_{j=1}^{4}\left(m\left(B\left(x_{\sigma(j)}\right)\right)-m\left(B\left(x_{\sigma(j-1)}\right)\right)\right) \underset{\mathbb{Q}_{i}}{\widetilde{A}_{i}^{(k)}}\left(x_{\sigma(j)}\right),
\end{array}
$$

where $u_{3}^{(1)} \geq u_{1}^{(1)} \geq u_{2}^{(1)} \geq u_{4}^{(1)}$, then $\underset{\otimes_{1}}{\widetilde{A}^{(1)}}\left(x_{\sigma(1)}\right)=\underset{\otimes_{1}}{\widetilde{A}^{(1)}}\left(x_{3}\right)$, $\underset{\otimes_{1}}{\widetilde{A}^{(1)}}\left(x_{\sigma(2)}\right)=\underset{\otimes_{1}}{\widetilde{A}^{(1)}}\left(x_{1}\right), \underset{\otimes_{1}}{\widetilde{A}^{(1)}}\left(x_{\sigma(3)}\right)=\underset{\otimes_{1}}{\widetilde{\widetilde{A}}^{(1)}}\left(x_{2}\right), \underset{\otimes_{1}}{\widetilde{A}^{(1)}}\left(x_{\sigma(4)}\right)=$ $\widetilde{A}_{\otimes 1}^{(1)}\left(x_{4}\right)$. We have

$$
\begin{gathered}
m\left(B\left(x_{\tau(1)}\right)\right)=m\left(\left\{x_{3}\right\}\right)=0.3, \\
m\left(B\left(x_{\tau(2)}\right)\right)=m\left(\left\{x_{1}, x_{3}\right\}\right)=0.6, \\
m\left(B\left(x_{\tau(3)}\right)\right)=m\left(\left\{x_{1}, x_{2}, x_{3}\right\}\right)=0.82, \\
m\left(B\left(x_{\tau(4)}\right)\right)=m\left(\left\{x_{1}, x_{2}, x_{3}, x_{4}\right\}\right)=1, \\
r_{1}^{(1)}=\text { I-IGULCOA }\left(\left\langle u_{1},{\widetilde{\otimes_{1}}}^{(1)}\left(x_{1}\right)\right\rangle,\left\langle u_{2}, \widetilde{A}_{\otimes_{1}}^{(1)}\left(x_{2}\right)\right\rangle,\right. \\
\left.\left\langle u_{3},{\widetilde{A_{1}}}^{(1)}\left(x_{3}\right)\right\rangle,\left\langle u_{4},{\widetilde{\otimes_{1}}}^{\widetilde{A}_{1}^{(1)}}\left(x_{4}\right)\right\rangle\right) \\
\left.=\bigoplus_{j=1}^{4}\left(m\left(B\left(x_{\sigma(j)}\right)\right)-m\left(B\left(x_{\sigma(j-1)}\right)\right)\right)\right)_{\otimes_{1}^{A}}^{\widetilde{A}_{1}^{(1)}}\left(x_{\sigma(j)}\right) \\
=\left(\left[s_{3.42}, s_{4.78}\right],[0.686,0.910]\right) .
\end{gathered}
$$

Similarly, we have

$$
\begin{aligned}
& r_{1}^{(1)}=\left(\left[s_{3.42}, s_{4.78}\right],[0.686,0.910]\right), \\
& r_{2}^{(1)}=\left(\left[s_{2.92}, s_{4.52}\right],[0.798,0.925]\right), \\
& r_{3}^{(1)}=\left(\left[s_{3.18}, s_{4.78}\right],[0.642,0.892]\right), \\
& r_{4}^{(1)}=\left(\left[s_{2.60}, s_{4.82}\right],[0.686,0.940]\right), \\
& r_{1}^{(2)}=\left(\left[s_{2.50}, s_{4.38}\right],[0.654,0.829]\right),
\end{aligned}
$$




$$
\begin{aligned}
& r_{2}^{(2)}=\left(\left[s_{2.94}, s_{4.34}\right],[0.686,0.892]\right), \\
& r_{3}^{(2)}=\left(\left[s_{2.22}, s_{3.52}\right],[0.686,0.849]\right), \\
& r_{4}^{(2)}=\left(\left[s_{2.72}, s_{4.52}\right],[0.765,0.925]\right), \\
& r_{1}^{(3)}=\left(\left[s_{3.20}, s_{4.40}\right],[0.731,0.853]\right), \\
& r_{2}^{(3)}=\left(\left[s_{2.62}, s_{4.42}\right],[0.603,0.849]\right), \\
& r_{3}^{(3)}=\left(\left[s_{2.64}, s_{4.02}\right],[0.597,0.853]\right), \\
& r_{4}^{(3)}=\left(\left[s_{2.62}, s_{4.82}\right],[0.546,0.824]\right) .
\end{aligned}
$$

Step 2. Utilize the decision information given in matrix $\widetilde{R}$ and the IGULCOA operator:

$$
\begin{aligned}
\widetilde{z}_{i} & =\operatorname{IGULCOA}\left(\widetilde{r}_{i}^{(1)}, \widetilde{r}_{i}^{(2)}, \widetilde{r}_{i}^{(3)}\right) \\
& =\bigoplus_{k=1}^{3}\left(\mu\left(B\left(d_{\tau(k)}\right)\right)-\mu\left(B\left(d_{\tau(k-1)}\right)\right)\right) \widetilde{r}_{\tau(i)}^{(k)} .
\end{aligned}
$$

We obtain the collective overall preference values $\widetilde{z}_{i}$ of the decision makers $D_{k}(k=1,2,3)$. Consider

$$
\begin{aligned}
& \widetilde{z}_{1}=\left(\left[s_{3.091}, s_{4.509}\right],[0.971,0.998]\right), \\
& \widetilde{z}_{2}=\left(\left[s_{2.834}, s_{4.454}\right],[0.975,0.999]\right), \\
& \widetilde{z}_{3}=\left(\left[s_{2.709}, s_{4.149}\right],[0.955,0.998]\right), \\
& \widetilde{z}_{4}=\left(\left[s_{2.648}, s_{4.715}\right],[0.967,0.999]\right) .
\end{aligned}
$$

Step 3. Let the basic unit-interval monotonic (BUM) function be $\rho(y)=y^{2}$. We get the size of the collective overall preference values $Q\left(\widetilde{z}_{i}\right)(i=1,2,3,4)$ by using

$$
\begin{aligned}
& Q\left(\widetilde{z}_{1}\right)=\left[s_{0.062}, s_{0.091}\right], \\
& Q\left(\widetilde{z}_{2}\right)=\left[s_{0.049}, s_{0.076}\right], \\
& Q\left(\widetilde{z}_{3}\right)=\left[s_{0.084}, s_{0.129}\right], \\
& Q\left(\widetilde{z}_{4}\right)=\left[s_{0.060}, s_{0.106}\right] .
\end{aligned}
$$

Step 4. To rank these collective overall preference values $\widetilde{\mathrm{Q}}\left(\widetilde{z}_{i}\right)(i=1,2,3,4)$ by using (29) and then construct a complementary matrix,

$$
P=\left[\begin{array}{llll}
0.500 & 0.752 & 0.095 & 0.415 \\
0.248 & 0.500 & 0.000 & 0.223 \\
0.905 & 1.000 & 0.500 & 0.755 \\
0.585 & 0.777 & 0.245 & 0.500
\end{array}\right]
$$

Summing all elements in each line of matrix $P$, we have

$$
\begin{array}{ll}
p_{1}=0.230, & p_{2}=0.164, \\
p_{3}=0.347, & p_{4}=0.259 .
\end{array}
$$

Then, we rank $\widetilde{Q}\left(\widetilde{z}_{i}\right)(i=1,2,3,4)$ in a descending order in accordance with the values of $p_{i}(i=1,2,3,4): \widetilde{Q}\left(\widetilde{z}_{3}\right)>$ $\widetilde{\mathrm{Q}}\left(\widetilde{z}_{4}\right)>\widetilde{\mathrm{Q}}\left(\widetilde{z}_{1}\right)>\widetilde{\mathrm{Q}}\left(\widetilde{z}_{2}\right)$, so we get the $\widetilde{z}_{3}>\widetilde{z}_{4}>\widetilde{z}_{1}>\widetilde{z}_{2}$.

Step 5. Rank all the alternatives $a_{i}(i=1,2,3,4)$ in accordance with $\widetilde{z}_{i}(i=1,2,3,4): a_{3}>a_{4}>a_{1}>a_{2}$. Thus, the best alternative is $a_{3}$.

\section{Conclusion}

In this paper, we use the Choquet integral to propose the interval grey uncertain linguistic correlated ordered arithmetic aggregation (IGULCOA) operator and the induced interval grey uncertain linguistic correlated ordered geometric aggregation (I-IGULCOA) operator, which are used to discuss the correlative interval grey uncertain linguistic variables. Furthermore, we also analyze the relation between it and some known operators and develop an approach to multiple attribute group decision making with the correlative attributes weights and the correlative expert weights, in which the attribute values are given in terms of the induced interval grey uncertain linguistic variables based on the interval grey uncertain linguistic correlated ordered arithmetic aggregation (IGULCOA) operator and the induced interval grey uncertain linguistic correlated ordered geometric aggregation (I-IGULCOA) operator. Finally, an illustrative example has been given to show the developed method. The applications of the operator in many actual fields, such as decision making, pattern recognition, and clustering analysis, are open questions for future research.

\section{Acknowledgments}

This research was supported by the National Natural Science Foundation of China (nos. 90924012, 71090402, 71103149); Program for New Century Excellent Talents in University (no. NCET-10-0706); Specialized Research Fund for the Doctoral Program of Higher Education (no. 20090184110029); Humanities and Social Science Research Foundation of the Ministry of Education (no. 08JC630067); Sichuan Youth Science and Technology Foundation (no. 09ZQ026-021); Fund for Cultivating Academic and Technological Leaders in Sichuan Province (no. 2011-441); Fundamental Research Funds for the Central Universities (no. SWJTU11CX152).

\section{References}

[1] L. A. Zadeh, "Fuzzy sets," Information and Control, vol. 8, pp. 338-353, 1965.

[2] D. Ju-Long, "Control problems of grey systems," Systems \& Control Letters, vol. 1, no. 5, pp. 288-294, 1982.

[3] J. Kacprzyk, "Group decision making with a fuzzy linguistic majority," Fuzzy Sets and Systems, vol. 18, no. 2, pp. 105-118, 1986.

[4] P. Liu and F. Jin, "The multi-attribute group decision making method based on the interval grey linguistic variables," African Journal of Business Management, vol. 4, no. 17, pp. 3708-3715, 2010.

[5] P. D. Liu and X. Zhang, "Multi-attribute group decision making method based on interval grey linguistic variables weighted 
geometric aggregation operator," Control and Decision, vol. 26, no. 5, pp. 743-747, 2011.

[6] Z. Su, G. Xia, M. Chen, and L. Wang, "Induced generalized intuitionistic fuzzy OWA operator for multi-attribute group decision making," Expert Systems with Applications, vol. 39, no. 2, pp. 1902-1910, 2012.

[7] Z. Yue, "A method for group decision-making based on determining weights of decision makers using TOPSIS," Applied Mathematical Modelling, vol. 35, no. 4, pp. 1926-1936, 2011.

[8] G. W. Wei, "FIOWHM operator and its application to multiple attribute group decision making," Expert Systems with Applications, vol. 38, no. 4, pp. 2984-2989, 2011.

[9] G. Wei, X. Zhao, R. Lin, and H. Wang, "Generalized triangular fuzzy correlated averaging operator and their application to multiple attribute decision making," Applied Mathematical Modelling, vol. 36, no. 7, pp. 2975-2982, 2012.

[10] Z. Xu, "Uncertain linguistic aggregation operators based approach to multiple attribute group decision making under uncertain linguistic environment," Information Sciences, vol. 168, no. 1-4, pp. 171-184, 2004.

[11] Z. Xu, "A note on linguistic hybrid arithmetic averaging operator in multiple attribute group decision making with linguistic information," Group Decision and Negotiation, vol. 15, no. 6, pp. 593-604, 2006.

[12] Z. Xu, "An approach based on the uncertain LOWG and induced uncertain LOWG operators to group decision making with uncertain multiplicative linguistic preference relations," Decision Support Systems, vol. 41, no. 2, pp. 488-499, 2006.

[13] J. M. Merigó and G. Wei, "Probabilistic aggregation operators and their application in uncertain multi-person decisionmaking," Technological and Economic Development of Economy, vol. 17, no. 2, pp. 335-351, 2011.

[14] D. W. Chen, Grey Fuzzy Set Introduction, Heilongjiang Science and Technology Press, Harbin, China, 1994.

[15] G. Z. Bu and Y. W. Zhang, "Grey fuzzy comprehensive evaluation based on the theory of grey fuzzy relation," Systems Engineering, vol. 4, no. 4, pp. 141-144, 2002.

[16] N. Jin and S. C. Lou, "Study on multi-attribute decision making model based on the theory of grey fuzzy relation," Intelligence Command Control and Simulation Techniques, vol. 7, pp. 44-47, 2003.

[17] N. Jin and S. Lou, "A grey fuzzy multi-attribute decision making method," Fire Control \& Command Control, vol. 4, pp. 26-28, 2004.

[18] L. Dang and L. Sifeng, "Analytic method to a kind of grey fuzzy decision making based on entropy," Engineering Science, vol. 10, pp. 48-51, 2004.

[19] K. Meng, Y. Li, C. Wang, and W. Yang, "Interval-value grey fuzzy comprehensive evaluation based on the preference of the risk and its application," Fire Control and Command Control, vol. 2007, no. 4, pp. 109-111, 2007.

[20] E. Cables, M. S. García-Cascales, and M. T. Lamata, "The LTOPSIS: an alternative to TOPSIS decision-making approach for linguistic variables," Expert Systems with Applications, vol. 39, no. 2, pp. 2119-2126, 2011.

[21] M. Delgado, J. L. Verdegay, and M. A. Vila, "Linguistic decisionmaking models," International Journal of Intelligent Systems, vol. 7, no. 5, pp. 479-492, 1992.

[22] F. Herrera, E. Herrera-Viedma, and J. L. Verdegay, "Direct approach processes in group decision making using linguistic OWA operators," Fuzzy Sets and Systems, vol. 79, no. 2, pp. 175190, 1996.
[23] F. Herrera and E. Herrera-Viedma, "Linguistic decision analysis: steps for solving decision problems under linguistic information," Fuzzy Sets and Systems, vol. 115, no. 1, pp. 67-82, 2000.

[24] J. M. Merigó, M. Casanovas, and L. Martínez, "Linguistic aggregation operators for linguistic decision making based on the Dempster-Shafer theory of evidence," International Journal of Uncertainty, Fuzziness and Knowledge-Based Systems, vol. 18, no. 3, pp. 287-301, 2010.

[25] Z. Xu, "Choquet integrals of weighted intuitionistic fuzzy information," Information Sciences, vol. 180, no. 5, pp. 726-736, 2010.

[26] S.-M. Zhou, F. Chiclana, R. I. John, and J. M. Garibaldi, “Type1 OWA operators for aggregating uncertain information with uncertain weights induced by type-2 linguistic quantifiers," Fuzzy Sets and Systems, vol. 159, no. 24, pp. 3281-3296, 2008.

[27] Y. H. Chen, T. C. Wang, and C. Y. Wu, "Multi-criteria decision making with fuzzy linguistic preference relations," Applied Mathematical Modelling, vol. 35, no. 3, pp. 1322-1330, 2011.

[28] N. Zhang and G. W. Wei, "Method for aggregating correlated interval grey linguistic variables and its application to decision making," Technological and Economic Development of Economy, vol. 19, no. 1, pp. 60-77, 2013.

[29] G. Choquet, “Theory of capacities," Annales de l'Institut Fourier, vol. 5, pp. 131-295, 1953.

[30] R. R. Yager, "Induced aggregation operators," Fuzzy Sets and Systems, vol. 137, no. 1, pp. 59-69, 2003.

[31] R. Yager, "OWA aggregation over a continuous interval argument with applications to decision making," IEEE Transactions on Systems Man and Cybernetics B, vol. 34, no. 5, pp. 1952-1963, 2004.

[32] C. Tan and X. Chen, "Induced Choquet ordered averaging operator and its application to group decision making," International Journal of Intelligent Systems, vol. 25, no. 1, pp. 59-82, 2010.

[33] G. W. Wei and X. Zhao, "Some induced correlated aggregating operators with intuitionistic fuzzy information and their application to multiple attribute group decision making," Expert Systems with Applications, vol. 39, no. 2, pp. 2026-2034, 2012.

[34] L. A. Zadeh, "The concept of a linguistic variable and its application to approximate reasoning-I," Information Sciences, vol. 8, no. 3, pp. 199-249, 1975.

[35] Z. Xu, "Deviation measures of linguistic preference relations in group decision making," Omega, vol. 33, no. 3, pp. 249-254, 2005.

[36] Z. Xu, "An interactive approach to multiple attribute group decision making with multigranular uncertain linguistic information," Group Decision and Negotiation, vol. 18, no. 2, pp. 119145, 2009.

[37] R. R. Yager, "Choquet aggregation using order inducing variables," International Journal of Uncertainty, Fuzziness and Knowledge-Based Systems, vol. 12, no. 1, pp. 69-88, 2004.

[38] Z. Y. Wang and G. J. Klir, Fuzzy Measure Theory, Plenum Publishing, New York, NY, USA, 1992. 


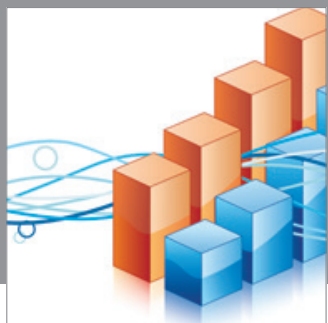

Advances in

Operations Research

mansans

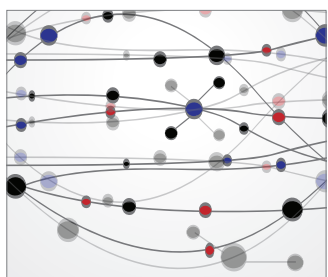

The Scientific World Journal
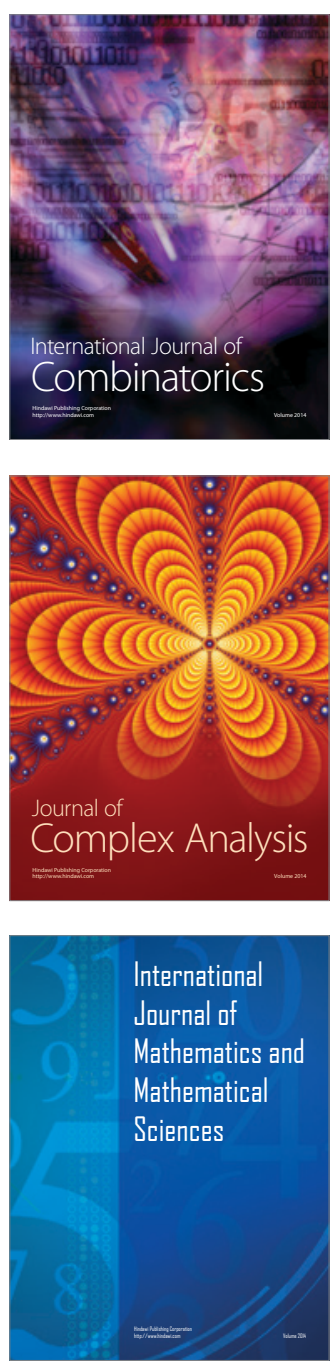
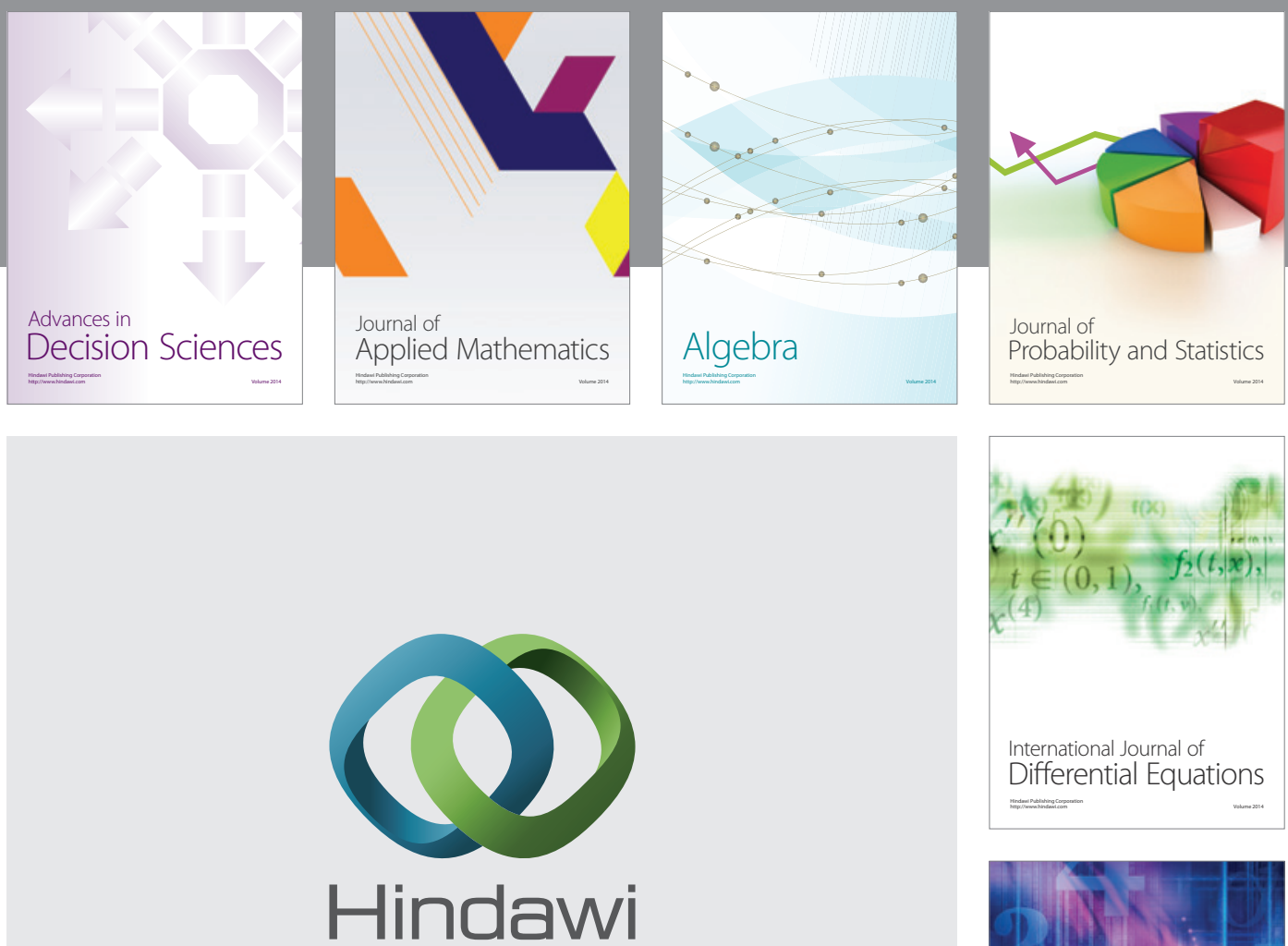

Submit your manuscripts at http://www.hindawi.com
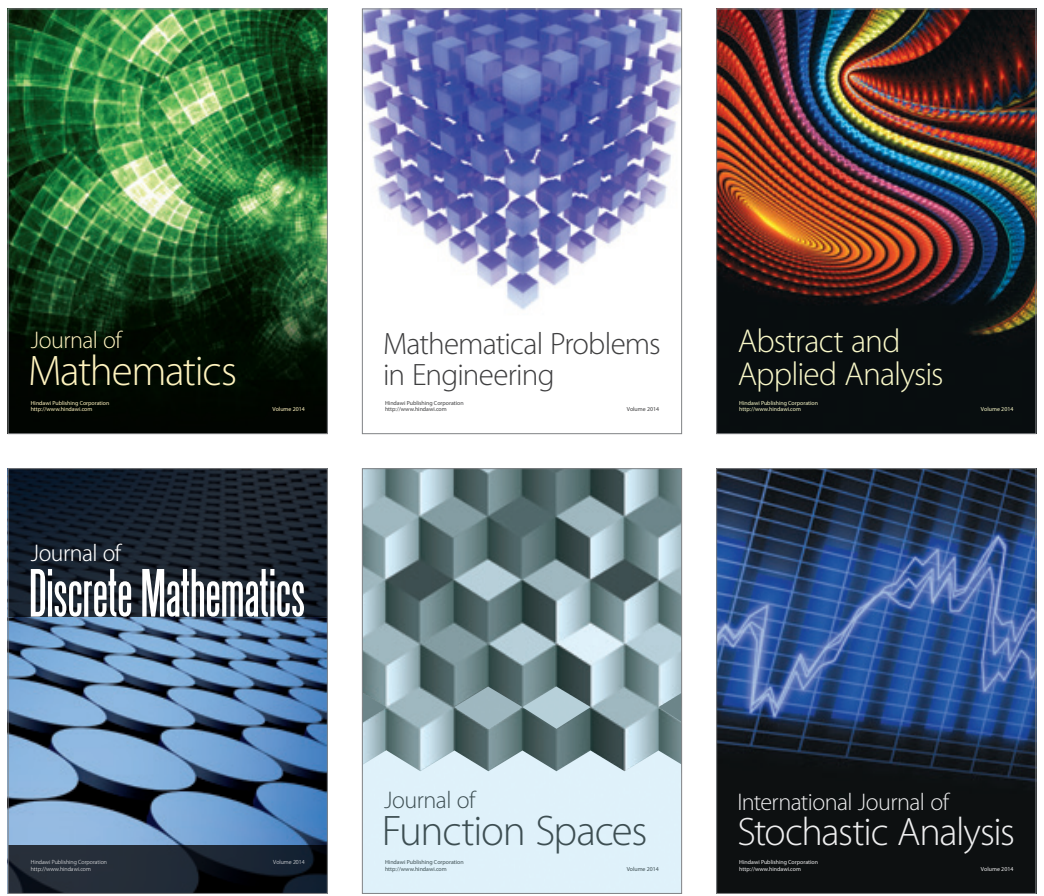

Journal of

Function Spaces

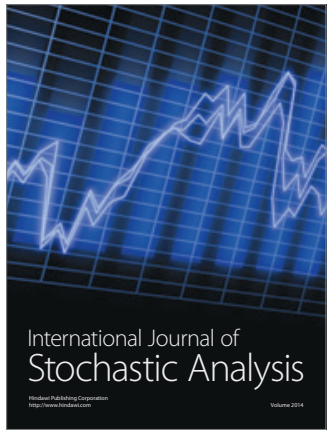

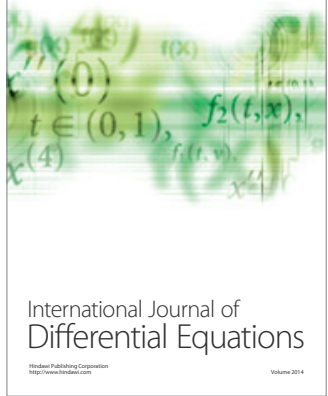
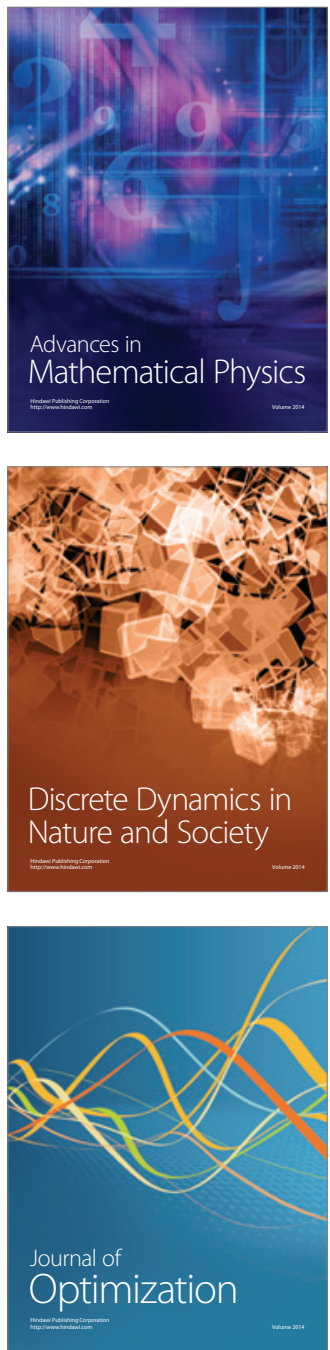